\title{
BUSINESS RESEARCH. WAS? WARUM? WOHIN?
}

\section{von Birgit Bauer}

Zusammenfassung: In den letzten Jahrzehnten der Globalisierung und des Internet-Booms hat sich die Wirtschaftsrecherche, oft auch als Business Research bezeichnet, etabliert. Nicht nur bei großen Konzernen, auch bei Unternehmensgründern oder kleinen und mittelständischen Unternehmen. Sie ist nicht mehr "Nice to have", sondern zur unternehmerischen Notwendigkeit geworden. Noch vor etwa zwei Jahrzehnten waren viele Informationen schwer zugänglich und ihre Beschaffung aufwändig und teuer. Heute steht uns durch die Digitalisierung und das Internet eine Flut von Informationen zur Verfügung. Was sind typische Fragen? Welche Informationsquellen werden genutzt? Wie hat sich die Wirtschaftsrecherche in den letzten Jahrzehnten verändert? Was sind aktuelle Entwicklungen?

Schlüsselwörter: Business Research; Business Information; Business Intelligence; Wirtschaftsrecherche; Marktrecherche; Information Professional; Informationbrokering; Info-Broker; Contentmarketing; Soziale Medien; Outsourcing; Off shoring

\section{BUSINESS RESEARCH. WHAT? WHY? WHERE TO?}

Abstract: In the last decades of globalization and the internet boom, business research has become established. Not only with big corporations, but also with company founders or small and medium-sized enterprises. It is not a "nice to have" but has become essential in today's business. Just two decades ago a lot of information was difficult and expensive to get. Today, digitalization and the internet are a rich source of information. What are typical questions? What sources of information get used? How has business research changed in recent decades? What are current developments?

Keywords: Business Research; Business Information; Business Intelligence; Market Research; Information Professional; Informationbrokering; Info-Broker; Content marketing; Social Media; Outsourcing; Off shoring

DOI: https://doi.org/10.31263/voebm.v71i3-4.2151

(c) Birgit Bauer

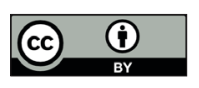

Dieses Werk ist lizenziert unter einer

Creative-Commons-Lizenz Namensnennung 4.0 International 


\section{Inhalt}

1. Was ist Business Research?

2. Warum Business Research?

3. Wie wird recherchiert?

4. Wohin zeigen aktuelle Trends?

\section{Was ist Business Research?}

In der Regel wird darunter die systematische Recherche, Analyse, Aufbereitung und Verbreitung relevanter Informationen und Erkenntnisse im Wirtschaftsbereich verstanden.

Business Research, Marktrecherche, Marktforschung oder Marketingforschung betreffen zum Teil dieselben Fragestellungen und nutzen teilweise dieselben Methoden. Daher wird zwischen diesen Begriffen oft nicht unterschieden. Unter Marktforschung versteht man meist die systematische Erhebung, Analyse und Interpretation von Informationen über Gegebenheiten und Entwicklungen auf Märkten (z.B. Absatz- und Beschaffungsmärkte), um relevante Informationen für Marketing-Entscheidungen bereitzustellen [1].

Für diesen Artikel ist mit Business Research jede Art der wirtschaftsbezogenen Informationsbeschaffung gemeint. Business Research geht also über den Begriff der reinen Marktforschung hinaus, wenn beispielsweise Fachzeitschriften technische Produktvergleiche für die eigenen Artikel beauftragen oder Unternehmen die Auswertung wissenschaftlicher Forschung.

Häufige Fragen in der Business Research-Praxis sind: Wie groß ist ein bestimmter Markt? Wer sind die Key Player, also die wichtigsten Firmen? Wie arbeiten und positionieren sich diese? Gibt es Neuigkeiten? Welche Produkte gibt es schon, mit welchen Eigenschaften, zu welchen Preisen? Welche Vertriebswege sind in der Branche typisch? Was sind aktuelle Trends?

Außerhalb des deutschen Sprachraums hat der Begriff Business Research eine zweite Bedeutung - die universitäre betriebswirtschaftliche Forschung -, diese ist im vorliegenden Artikel nicht gemeint.

\section{Warum Business Research?}

Wirtschaftsrecherchen werden durchgeführt, um wirtschaftlich erfolgreiche Entscheidungen zu ermöglichen. Große Firmen beobachten ihre Märkte und MitbewerberInnen kontinuierlich. Kleinere Firmen oder Unter- 
nehmensgründerInnen recherchieren zumindest dann, wenn wichtige Entscheidungen anstehen. Zum Beispiel, wenn sie in neue Märkte expandieren oder neue Produkte auf den Markt bringen wollen, wenn sie neue Anwendungen für ihr Verfahren oder ihre Technologie suchen oder aber die Bank und andere InvestorInnen von ihrem Konzept überzeugen möchten.

\section{Wie wird recherchiert?}

Trotz der Informationsfülle im Internet kommt in der professionellen Wirtschaftsrecherche nicht nur Google zum Einsatz. Nach wie vor sind kommerzielle Datenbanken eine wichtige Quelle. Fast jedes Projekt beinhaltet auch Telefonate. Die Ergebnisse setzen sich wie Puzzle aus unterschiedlichen Quellen kommenden, vielen einzelnen Informationen zusammen. Immer wichtiger wird die rasche und effiziente Recherche, die kritische Auswahl der Inhalte, aber auch die Aufbereitung der Ergebnisse in kundenfreundlicher, leicht und schnell erfassbarer Form.

Je nach Aufgabenstellung kommen unterschiedliche Methoden und Informationsquellen zum Einsatz. Zwei Beispiele aus meiner Recherche-Praxis veranschaulichen auch die Bandbreite der Themen und Projekte:

- Im Zuge der Unternehmensgründung der Konditorei CupCakes beauftragte die Gründerin eine Marktanalyse zum Geschäftszweig Design-Hochzeitstorten. Zuerst prüfte ich, ob es schon fertige Marktstudien zu diesem Thema gibt. Leider nein. Dann grenzte ich die Marktgröße durch statistisches Material ein: Wie viele Hochzeiten werden in der Umgebung geschlossen? Demografische und finanzielle Daten der in Frage kommenden Bezirke halfen bei der Abschätzung, wie viele Paare an edlen Design-Torten interessiert sein könnten. Anhand eines gemeinsam skizzierten Fragebogens befragte die Unternehmerin Hochzeitsplanerinnen und Bräute auf Brautmessen. Auch damit wurde deutlich, welcher Anteil an Brautpaaren eine Hochzeitstorte kaufen würde. Die Konkurrenzanalyse - wer bietet sonst noch Torten in anspruchsvollem Design und Premium-Ausführung an und zu welchem Preis - vervollständigte die Marktanalyse. Der Abschlussbericht unterstützte die Gründerin bei ihren Entscheidungen und half bei den Gesprächen mit der Bank. Der Betrieb hat sich sehr erfolgreich entwickelt. Schon kurz nach der Gründung konnte die Unternehmerin zwei weitere Filialen an prominenten Standorten in Wien eröffnen. 
- Das zweite Fallbeispiel stammt aus dem High-Tech- und Wissenschaftsbereich. Für den EU-Förderantrag des Austrian Institute of Technology (AIT) zur Quantenkryptographie, also zur Verschlüsselung in der Nachrichtenübertragung, - das klang damals noch nach „Science-Fiction“ -, recherchierte ich in (sehr teuren) kommerziellen Datenbanken. Im World Wide Web hatte das AIT bereits selbst intensiv gesucht. Ich konnte eine aktuelle Studie eines renommierten, internationalen Marktforschungsinstitutes finden, in der die Quantenverschlüsselung als eine potenzielle Konkurrenztechnologie bewertet wurde. Interviews mit internationalen ExpertInnen, die das in Zukunft mögliche kommerzielle Potenzial kommentierten, ergänzten die Recherche. Auch aufgrund dieser Prognosen konnten 11 Millionen Euro an EU-Fördermitteln lukriert werden. 2016 war Österreich bei der Vorbereitung einer neuen Flagship-Initiative der EU, für Quantentechnologie, abermals führend beteiligt.

\section{Wohin zeigen aktuelle Trends?}

Die Entwicklungen im Informationsbereich der letzten zwei Jahrzehnte waren schon rasant. Erinnern wir uns zurück: Anfang 2000 hatte in Österreich nur $11 \%$ der Bevölkerung zu Hause einen Internetzugang, Firmen nutzten digital vor allem eMail-Dienste, Yahoo war das populärste Suchwerkzeug weltweit, Google war noch fast unbekannt, es gab kein Facebook und keine Wikipedia. Heute steht uns durch die Digitalisierung und das Internet eine Flut an Daten und Informationen zur Verfügung. Suchen wir aktuell „die Nadel im Heuhaufen“, waren wir früher oft froh, online überhaupt „den Heuhaufen zu finden“. Auch jetzt sind neue Entwicklungen erkennbar, wie zum Beispiel:

\section{- Content explodiert:}

Immer mehr Medien sind heute online verfügbar. Darunter Zeitungen, Magazine, Hörfunk, Fernsehen, oft auch mit Teilen ihrer Archive. Auch die Digitalisierungsprojekte der Bibliotheken tragen dazu bei. Die im Business Research ebenfalls genutzten kommerziellen Datenbanken gibt es nach wie vor. Sie punkten mit spezialisierten Inhalten und interessanten Funktionalitäten. Ihr Markt war zwar nach der Finanzkrise 2008 schwierig, wächst aber, wie internationale Studien belegen (u.a. Outsell 2015 [2], Clearwater International 2016 [3], McKinsey 2009 [4]). 
Content Marketing ist ein aktueller Trend, mit steigender Tendenz. Dabei handelt es sich um eine Marketing-Technik, die mit informierenden, beratenden und unterhaltenden Inhalten die Zielgruppe ansprechen und zu gewinnbringendem Kundenverhalten anregen soll - im Gegensatz zu reiner Werbung, in der die positive Darstellung des Produkts oder des Unternehmens im Mittelpunkt steht. Das Dr. Oetker-Backbuch für die Vermarktung des Backpulvers ist ein bekanntes und historisches Content Marketing-Beispiel. Mehr und mehr Firmen machen durch themenverwandte Artikel, Blogs, Newsletter, Whitepapers, Studien, Webinare und weitere Formate auf sich und ihre Produkte aufmerksam. Diese persönliche Beobachtung wird auch durch internationale Berichte bestätigt (u.a. HubSpot/smartInsights 2015 [5] und Ascend2 2015 [6]). Viele der Artikel oder Studien sind informativ und professionell gemacht. In der Wirtschaftsrecherche sind dadurch Statistiken, Analysen, Markteinschätzungen oder weiterführende Ideen überhaupt und noch dazu schnell verfügbar. Man kann nicht von der Objektivität oder Zuverlässigkeit dieser Informationen ausgehen und muss sie somit kritisch hinterfragen. Dennoch kann dieser Content ein guter Start in verschiedene Themen sein.

Die immer populäreren Sozialen Medien sind ebenfalls ein reicher Fundus an Meinungen - unter anderem über MitbewerberInnen oder Produkte, an neuen Schlagworten, Trends oder Kontakten. Außerdem gibt es bereits eine Reihe von Werkzeugen, Soziale Medien zu durchsuchen, zu analysieren und für die Wirtschaftsrecherche zu nutzen, wie beispielsweise Hootsuite, SocialMention, TweetReach oder Iconosquare.

Das heißt für Business Research: Die praktische Arbeit verändert sich durch diese digitale Informationsflut. Einerseits ist es leichter geworden, zu einer Fragestellung schnell verfügbare und relevante Informationen zu finden. Andererseits heißt es, umso mehr kritisch zu hinterfragen: Wer hat diesen Artikel, diese Studie, dieses Webinar erstellt, wann und aus welchem Grund? Warum ist diese Information online? Passt sie zu anderen Ergebnissen? Warum, oder warum nicht? Professionelle Sorgfalt und Expertise sind gefragt.

\section{- Outsourcing wächst stetig:}

Outsourcing, das Auslagern von Unternehmensaufgaben und -strukturen an externe Dienstleisterlnnen, hat in den letzten Jahrzehnten der Digitalisierung ein starkes Wachstum gezeigt. Wurden vor ein paar Jahrzenten vor allem IT- und Callcenter-Leistungen outgesourced, gilt dies heute zunehmend auch für wissensintensive Leistungen, wie 
Research \& Analytics. Outgesourced wird entweder ins Inland oder aber ins Ausland, dann spricht man auch von Off shoring. Indien ist im Outsourcing und Off shoring weltweit führend. Zur langjährigen Erfahrung kommen "gereifte“ Prozesse bei den AnbieterInnen, vielfältige Leistungen und Geschäftsmodelle, ein großer englischsprachiger „talent pool“, niedrige Löhne und mehr. Auch andere Länder, wie zum Beispiel China, Malaysia, Indonesien, die Philippinen oder Polen, sehen die Vorteile dieser Branche und ziehen nach. USA, Kanada, Westeuropa, Australien und Japan sind die wichtigsten Kundenmärkte. Das Outsourcing wächst allgemein auch was Informations-, Wissens- und Rechercheservices betrifft, wie internationale Studien zeigen (u.a. Technavio 2015 [7], Deloitte 2014 [8], Foster 2015 [9]). Gerade global aktive Firmen lagern zum Teil ganze Teams - auch Business Intelligence-Teams - im eigenen Land oder ins billigere Ausland aus (u.a. Foster 2015 [9], Bauer 2010/2008 [10,11]).

Das heißt für Business Research: Einerseits ist Outsourcing zu einer ernstzunehmenden Konkurrenz für Informationsdienstleisterlnnen geworden. Für Business Research-ExpertInnen ergibt sich aber auch die Möglichkeit, Outsourcing selbst zu nutzen und damit ungeliebte Aufgaben abzugeben, Arbeitsspitzen abzufedern oder ergänzende Expertise flexibel und kurzfristig zuzukaufen.

\section{- Und noch mehr Entwicklungen:}

Die technischen Entwicklungen treiben weitere Veränderungen in der Informationsindustrie voran. Zum Beispiel:

- Mit der Popularität des Internet, den eigenen „Google“-Erfahrungen von jedermann - Information ist überall, jederzeit, mit jedem Gerät, sofort und meist kostenlos verfügbar - steigen auch die Erwartungen der Kunden, wenn sie sich an Informationsanbieter und Information Professionals wenden.

- Die Personalisierung von Informationen und Nachrichtenströmen durch Suchmaschinen, Medien und Werbung, also die Anpassung von Informationen und Diensten an die persönlichen Vorlieben, Bedürfnisse und Fähigkeiten der Benutzer, verändert den Zugang zu Informationen.

$\checkmark$ Big Data - riesige, komplexe und wenig strukturierte Datenmengen, die in der Vergangenheit mit den herkömmlichen Datenverarbeitungsmethoden nicht ausgewertet werden konnten (zum Beispiel Daten aus Interaktionen in Social Media, von Kunden- oder Bankkarten, von elektronischer Kommunikation) - können heute ma- 
schinell verarbeitet und analysiert werden. Große Firmen nutzen Big Data bereits für ihre Entscheidungen (siehe [2], Dow Jones 2016 [12], pwc 2016 [13]).

$\square$ Auch die Künstliche Intelligenz ist in der Wirtschaft zur Realität geworden und wird die Informationsindustrie und die Wirtschaftsrecherche verändern ([2], [12]).

Das heißt für Business Research: Es gilt, auf die Entwicklungen zu achten, neue smarte Tools zu nützen und die eigenen Leistungen weiterzuentwickeln.

Vieles ist in Bewegung. Wir werden sehen, was sich im Bereich Business Research durchsetzen wird.

\author{
Dipl.-Ing. ${ }^{\text {in }}$ Birgit Bauer \\ Bauer Business Research e.U. \\ E-Mail: office@bauerbusinessresearch.com
}

\title{
Literatur
}

Alle online verfügbaren Quellen wurden in der Zeit von Juni bis September 2016 abgefragt.

* Der vorliegende Artikel entstand als Follow-up zum Vortrag auf der ODOK-Konferenz im Herbst 2016 in Eisenstadt. Er ist nach wie vor aktuell.

1 Hans Christian Weis, Peter Steinmetz, „Marktforschung“, 5. überarbeitete und aktualisierte Auflage aus 2002, Friedrich Kiehl Verlag GmbH, Ludwigshafen, ISBN 347042525.

2 Outsell, "Tipping Point - Information Industry Outlook 2016", Oktober 2015, unter http://info.outsellinc.com/rs/422-MBV-091/images/ Outsell_CEO_Topics_01oct2015_Info_Industry_Outlook_2016_Tipping_Point.pdf

3 Clearwater International, "B2B Information and Database Services Clearthought", 2016, unter http://clearwaterinternational.com/library/b2b-information-and-database-services-clearthought-2016/

4 McKinsey, "B2B Information Services - The Opportunity for Strategic Growth", Working Paper, 2009, unter https://www.mckinsey.com/ / media/mckinsey/dotcom/client_service/media\%20and\%20entertainment/pdfs/b2b_information_services.ashx 
5 HubSpot and Smart Insights, „Driving Content Marketing Success in Europe“, 2015, unter http://cdn2.hubspot.net/hubfs/53/Driving Content_Marketing_2015.pdf

6 Ascend2, "Content Marketing Trends - Survey Summary Report", 2015, unter http://ascend2.com/home/wp-content/uploads/Content-Marketing-Trends-Summary-Report-150310.pdf

7 TechNavio, "Global Knowledge Process Outsourcing Market 20152019", November 2015, unter http://www.technavio.com/report/ global-ito-and-bpo-knowledge-process-outsourcing-market

8 Deloitte, "Deloitte's 2014 Global Outsourcing and Insourcing Survey", Dezember 2014, unter https://www2.deloitte.com/content/dam/ Deloitte/us/Documents/strategy/us-2014-global-outsourcing-insourcing-survey-report-123114.pdf

9 Allan Foster, "'Moving the corporateneedle'-Howarewe doing? The 2015 Business Information Survey", Business Information Review, Volume 32 Number 1, März 2015. https://doi.org/10.1177/0266382115581467

10 Birgit Bauer, "Outsourcing of Information Services to India: Challenges and Chances for Western Information Professionals", Vortrag auf der Online Information-Konferenz, Olympia London, 2010.

11 Birgit Bauer, Marc Vollenweider, "The Future of Knowledge Process Outsourcing" Vortrag auf der Offshoring \& Outsourcing Conference SDA Bocconi, Mailand, 2008.

12 InfoDesk/Dow Jones "2020 Vision - The Future of Business Information”, White paper, 2016, unter http://www.infodesk.com/2020-vision-whitepaper

13 PricewaterhouseCoopers (pwc) "Business of Evicence 2016", White paper, 2016, unter https://www.pwc.co.uk/assets/pdf/business-ofevidence-report.pdf 